\title{
Determination of dynamic stress in fibre reinforced materials under impact load
}

\author{
D.H. Müller, Th. Franz and A. Tenzler \\ Bremer Institut für Konstruktionstechnik (BIK), \\ University of Bremen, 28359 Bremen, Germany \\ Tel.: +49421 2184262; Fax: +49421 2184263; \\ E-mail:bik@bik.uni-bremen.de
}

Received 5 December 1995

Revised 27 July 1998

The investigations reported in this paper are intended to obtain knowledge about stress concentrations within fibre reinforced plastics due to impact loadings below the damage threshold of the anisotropic material. The paper focuses on presenting the application of a dynamic photoelastic technique more than on providing extensive and detailed experimental data. Thus, a brief summary of the experimental part is given. The potential value of the developed experimental method is demonstrated exemplary for an arbitrary composite.

\section{Introduction}

The mechanical properties and the behaviour of fibre-reinforced plastic (FRP) have been studied especially for static loading of machine parts. The problem of determining the distribution of stresses and/or strains in a two-dimensional FRP-specimen subjected to an impact type of loading is difficult to solve mathematically. Therefore, experimental means are essential and, sometimes, indispensable. Fibre-reinforced composites are mainly used when high accelerations and velocities take place. Then, composites offer a high potential to reduce the kinetic energy. As a consequence, these parts very often have a high impact endangering. The reinforcement of the composites is a major parameter to reduce the material stress not only for static loadings but also when impact loadings take place. In the case of an impact loading, the viscoelasticity of the plastic matrix has to be considered. The photoelasticity offers an appropriate method for the investigation of impact stresses in reinforced materials, especially for stress concentrations due to complex shapes like notches or holes.

\section{Experimental impact simulation}

There are big differences regarding the stresses between static, quasi-static and impact loading. An impact is effected when the contact time is short compared to the time of wave pass through the part or specimen. If an impact loading is subjected to a structure, it is accelerated with the consequence that inertia forces are produced. Therefore, there is no linearity between the impact force and the stresses.

The experimental simulation of an impact requires the control of three characteristics: impact force $F(t)$, contact time and impulse. Within the loading device (Fig. 1), the impact is created by a steel projectile pneumatically accelerated in a pipe. The projectile velocity can be changed by altering the pneumatic pressure. A typical impact force history of the loadings applied during the experiments reported here is shown in Fig. 2. The contact time of about 10 to $20 \mu$ s is reasonable short to generate impact waves.

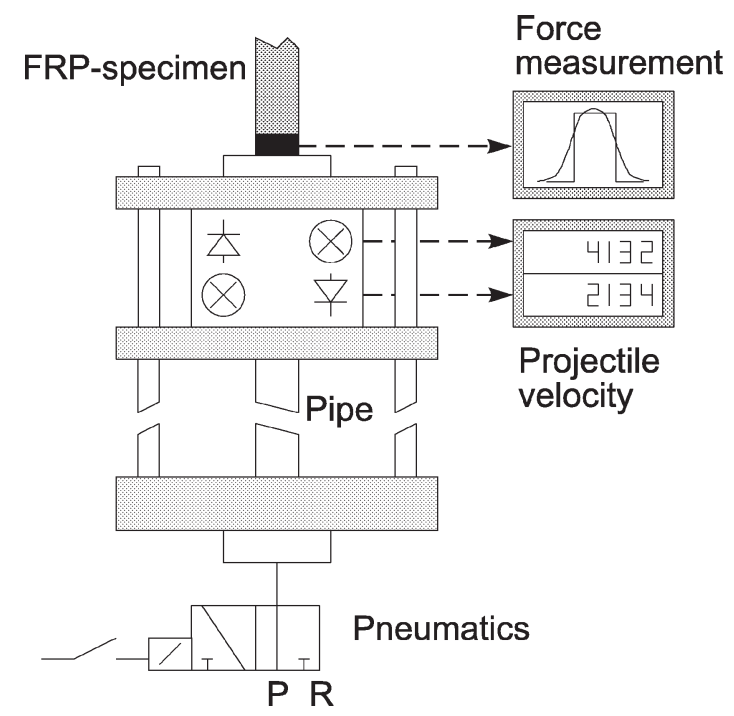

Fig. 1. Loading device. 


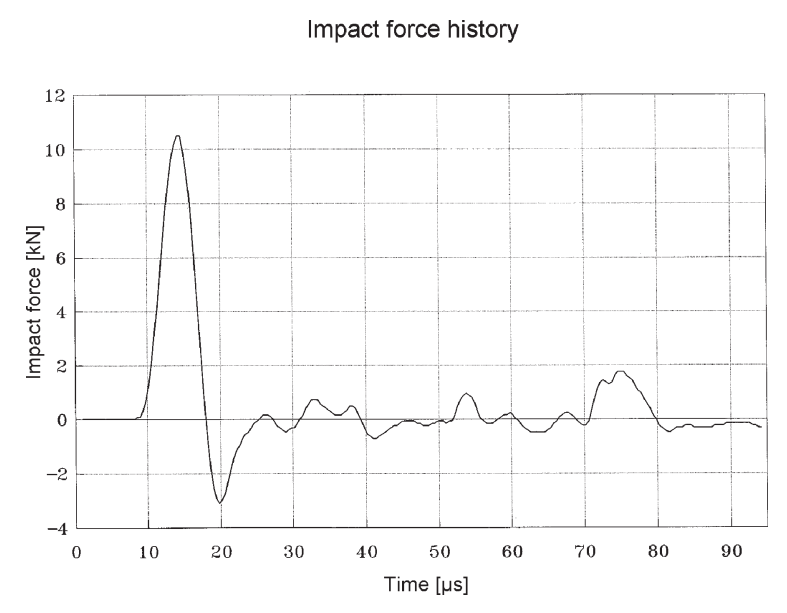

Fig. 2. Impact force history.

\section{Experimental analysis of stress concentration}

The stress analysis along a notch or hole contour requires a whole-field stress analysis method. In addition, the investigation of wave propagation due to impact loadings requires a technique with high time resolution. Therefore, the photoelastic stress coating method is employed in a single-flash operation mode. The experimental set-up derived from the reflection polariscope is depicted in Fig. 3. The exposure time is fixed to $500 \mathrm{~ns}$ by the Xenon flash-lights. Using a retarder, the delay of the shot time after the impact loading can be chosen independently in steps of $1 \mu \mathrm{s}$. The reproducibility of both the loading and the optical setup was proved in preliminary tests. The experimental technique has been described detailed elsewhere [3].

The unidirectional reinforcement was used as a standard [2] for the determination of material characteristics. The material investigated were unidirectional glass-fibre reinforced epoxy resin (GRP). Because of the fibre reinforcement, it is not possible to use the photoelastic effect of a birefringent matrix resin. Thus, a photoelastic coating is bonded to the composite with a reflecting cement. The specimens were $200 \mathrm{~mm} \times 200 \mathrm{~mm} \times 3 \mathrm{~mm}$ with an centric circular opening of $16 \mathrm{~mm}$. The stress coating were PS-1B (Measurements Group), $1.96 \mathrm{~mm}$, thick with a fringe constant $f=980 \mu \varepsilon /$ fringe. The specimen geometry is shown in Fig. 4.

The strains of the specimen are transmitted via the bonded surface to the photoelastic coating. When the circularly polarised light passes the coating, it is reflected by the cement or reflecting backing of the coating, and passes the coating a second time. The isochro-

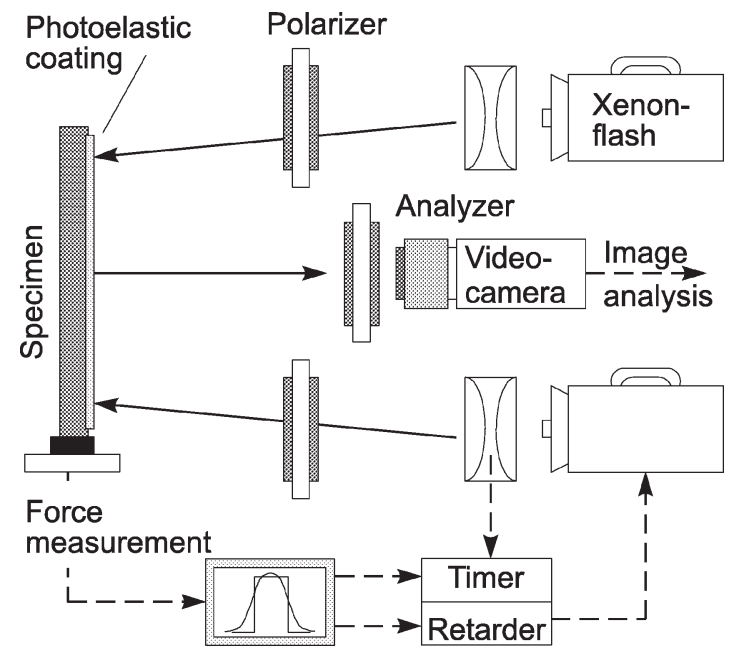

Fig. 3. Experimental set-up.

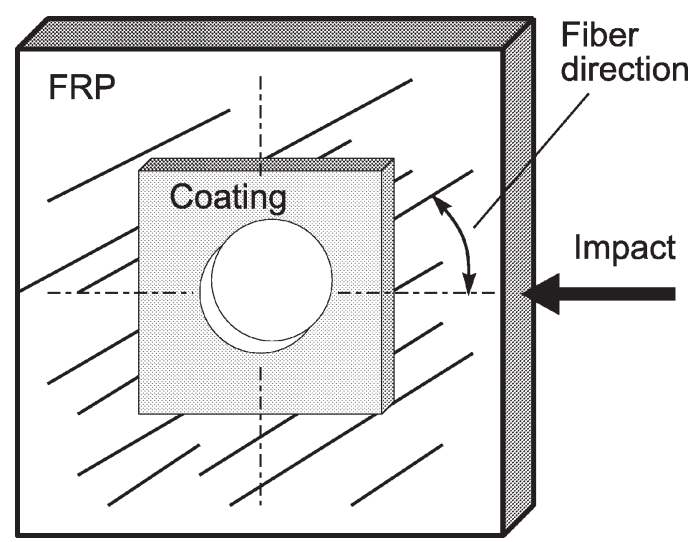

Fig. 4. FRP-specimen.

matic fringes increase linearly with the deformation of the specimen. The fringe pattern is recorded with a CCD-video camera. The image information of the coloured isochromatic fringes is transferred to a frame capture board of a computer. The fringe order along the notch edge can be automatically calculated using a digital image processing developed to specify the isochromatic order using the fringe colours [4].

Figure 5 shows the isochromatic fringe patterns of the notch area of two specimens. The left image was recorded $34 \mu$ s after the impact from a specimen with fibre reinforcement parallel with the impact direction. The right image was recorded $59 \mu$ s after the impact from a specimen with a reinforcement orientation of $45^{\circ}$ to the impact direction.

If the reinforcement and the impact direction are congruent the Young's modulus in impact direction and the propagation velocity of the impact wave are 


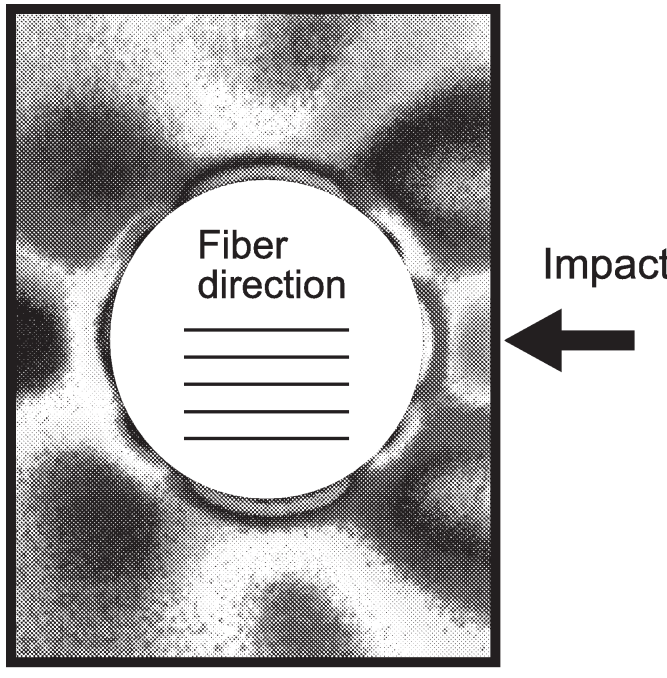

Time: $34 \mu \mathrm{s}$

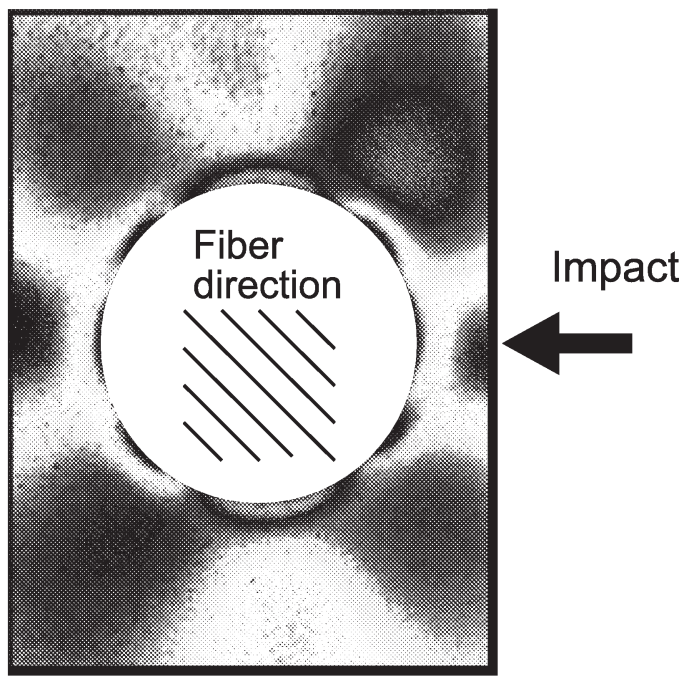

Time: $59 \mu \mathrm{s}$

Fig. 5. Isochromatic fringe pattern in a GRP disc using white light circular polariscope at $34 \mu$ s and $59 \mu$ s after impact.

maximum. Therefore, the stress maximum will reach the notch earlier for parallel orientation of impact and reinforcement than for nonparallel orientation.

The stress coating method allows the investigation of original parts with the disadvantage of the influences of the coating. Mainly, these are the increase of the specimen stiffness and the edge effect due to the mismatch of Poisson's ratios of specimen and coating. The ratio of stiffness change is dependent on the thickness, Young's modulus, and Poisson's ratio of specimen and coating, respectively. The stiffness change can be compensated by introducing a correction factor $K_{\mathrm{xy}}$, see Eq. (1):

$$
K_{\mathrm{xy}}=1+\frac{h_{\mathrm{s}}}{h_{\mathrm{xy}}} \cdot \frac{E_{\mathrm{s}}}{E_{\mathrm{xy}}} \cdot \frac{1+\nu_{\mathrm{xy}}}{1+\nu_{\mathrm{s}}},
$$

where $E_{\mathrm{s}}, E_{\mathrm{xy}}$ - moduli of coating and specimen, respectively; $h_{\mathrm{s}}, h_{\mathrm{xy}}$ - thickness of coating and specimen, respectively; $\nu_{\mathrm{s}}, \nu_{\mathrm{xy}}-$ Poisson's ratio of coating and specimen, respectively.

The coating influence due to the mismatch of Poisson's ratios is corrected by introducing another factor $K_{\nu}[3]$.

The strain of the pure composite can be calculated from the strain of the coating with Eq. (2):

$$
\Delta \varepsilon_{\mathrm{xy}}=K_{\mathrm{xy}} \cdot K_{\nu} \cdot \Delta \varepsilon_{\mathrm{s}}
$$

$\Delta \varepsilon_{\mathrm{s}}, \Delta \varepsilon_{\mathrm{xy}}-$ principal strain difference of coating and specimen, respectively.
Table 1

Material properties of coating and composite

\begin{tabular}{ccccc}
\hline Material & $\nu_{\text {stat }}$ & $\nu_{\text {imp }}$ & $E_{\text {stat }}(\mathrm{MPa})$ & $E_{\text {imp }}(\mathrm{MPa})$ \\
\hline PS-1B & 0.38 & 0.38 & 2450 & 2447 \\
GRP - 0 & 0.26 & 0.41 & 47,600 & 44,400 \\
GRP - 90 & 0.06 & 0.13 & 12,500 & 14,300 \\
\hline
\end{tabular}

To get the complete information about the stresses at the notch edge, it is essential to know the material properties according to the directions of the principal stresses. Since the Young's modulus under real impact condition is higher than under static loading, the material characteristics of coating and composite are determined experimentally from the velocity of longitudinal waves in bar-specimens of specific reinforcement orientations of $0^{\circ}, 45^{\circ}$ and $90^{\circ}$. Subsequent, the directiondepending elastic properties of the composite are calculated from these experimental results. The material properties of composite and coating for static and impact loading are tabulated in Table 1.

Within composites directions of the principal stress differs from direction the principal strain for off-axis loading or stress, respectively. As the photoelasticity coating method determines the principal strain it is necessary to calculate the angle between the both principal directions. Under consideration of these parameters the stress concentration can be calculated based on the fringe order according to the simplified Eq. (3) $[1,3]$ : 


$$
\sigma_{1}=\frac{K_{\nu} \cdot K_{\mathrm{xy}} \cdot f \cdot N}{\left(1+\nu_{\mathrm{xy}}\right) \cdot \sqrt{\left(S_{11}^{\prime}-S_{12}^{\prime}\right)^{2}+S_{16}^{\prime 2}}}
$$

with

$$
\begin{aligned}
S_{11}^{\prime}= & \frac{1}{E_{\mathrm{xy}}}, \quad S_{12}^{\prime}=\frac{\nu_{\mathrm{xy}}}{E_{\mathrm{xy}}}, \\
S_{16}^{\prime}= & \left(\cos ^{3} \theta \cdot \sin \theta-\sin ^{2} \theta \cdot \cos \theta\right) \\
& \times\left(\frac{2}{E_{\mathrm{L}}}+\frac{2 \nu_{\mathrm{LT}}}{E_{\mathrm{T}}}-\frac{1}{G_{\mathrm{LT}}}\right),
\end{aligned}
$$

where $\sigma_{1}$ - principal stress at the notch edge, $N-$ fringe order, $f$ - fringe constant of the stress coating, $E_{\mathrm{xy}}-$ Young's modulus in principal stress direction, $E_{\mathrm{L}}, E_{\mathrm{T}}-$ Young's modulus in and perpendicular to the reinforcement direction, respectively, $\nu_{\mathrm{xy}}, \nu_{\mathrm{LT}}-$ Poisson's ratio in principal stress and reinforcement direction, respectively, $G_{\mathrm{LT}}$ - shear modulus in reinforcement direction, $\theta$ - angle between reinforcement direction and principal stress direction.

\section{Stress along the hole contour}

Figures 6(a) and (b) show the calculated stresses along the hole edge of the two-dimensional specimen of Fig. 2. When the fibre direction is $0^{\circ}$, Fig. 6(a), there is a symmetrical behaviour of the stress field. The maximum stress appears $28 \mu$ s after the impact loading.

The example of Fig. 6(b) shows a fibre orientation of $45^{\circ}$. Here, the stress along the hole contour is asym-
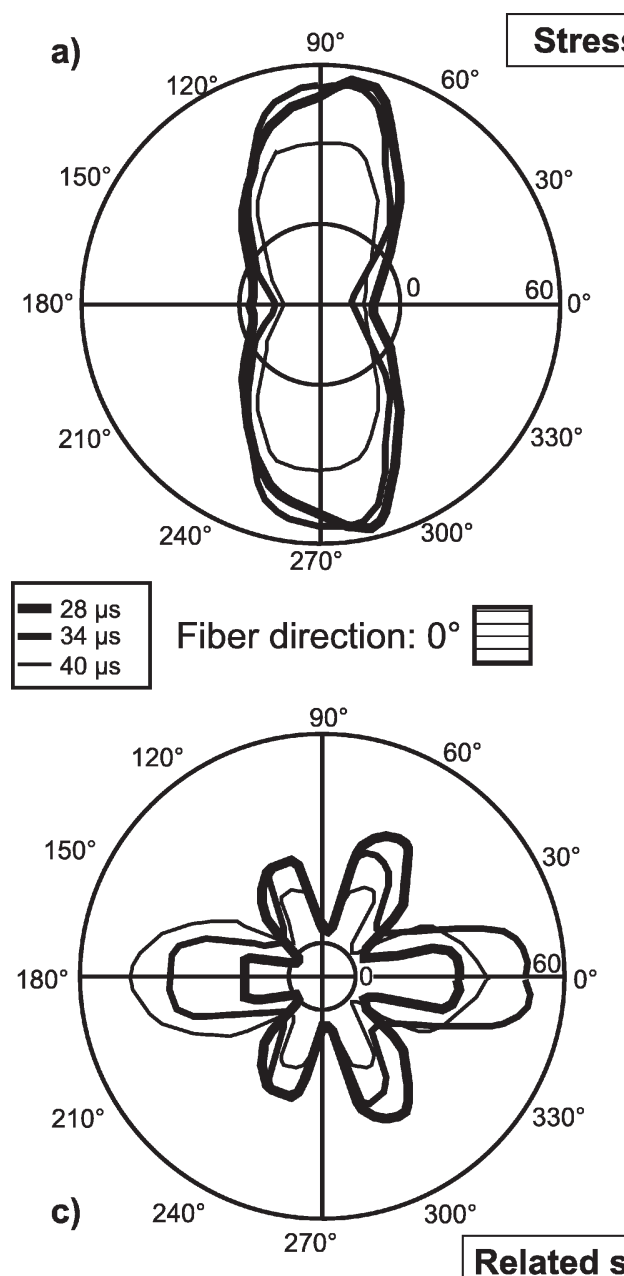

Related stress [\%]

Fig. 6. Stress and related stress along the hole contour of the specimen.
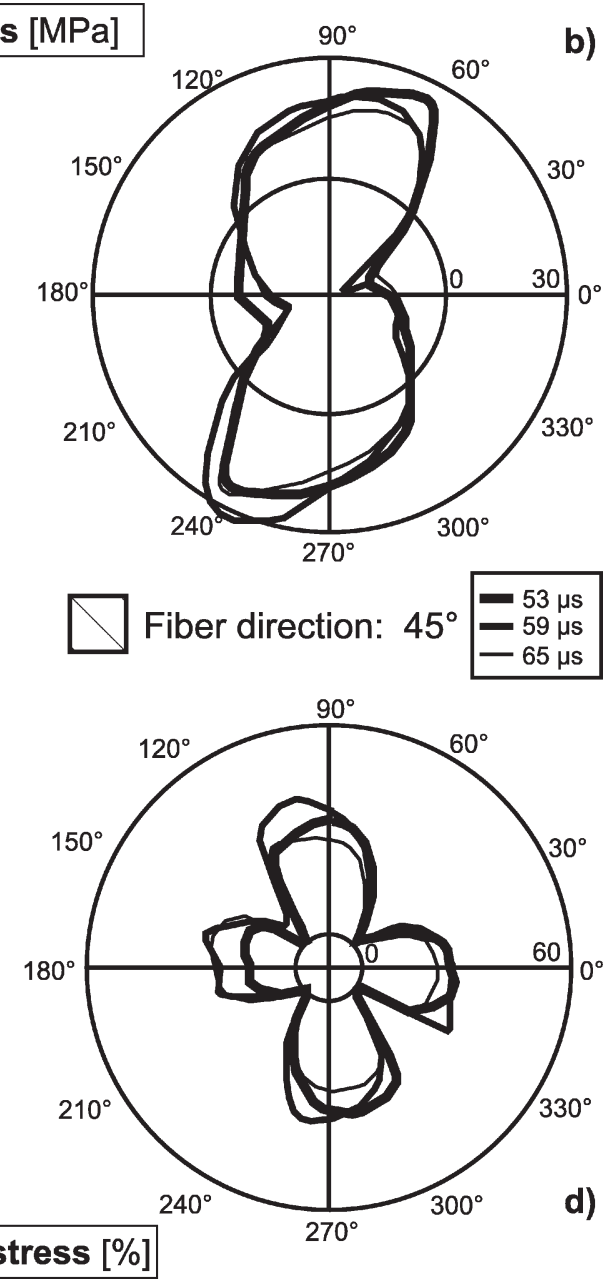

d) 
metrically. The time dependency of the stresses is similar to that of the fibre direction of $0^{\circ}$.

Comparing both diagrams it can be stated that the reinforcement direction largely influences the shock wave propagation, especially the maximum propagation velocity and the maximum stress. When the fibre reinforcement is orientated under $45^{\circ}$ to the impact direction the time dependency is much lower.

In dimensioning parts, it has usually to be assured that the internal stress does not exceed the strength of the material to resist failure. When the used material is anisotropic the strength depends on the direction. Therefore, the notch stress calculated with Eq. (3) has to be compared with the direction-depending strength of the composite.

Regarding the co-ordinate system along the hole contour as shown in Fig. 7, the 1-dimensional stress $\sigma_{1}$ along the notch edge can be transformed to the direction of the orthotropical axes with Eqs (4) to (6):

$$
\begin{aligned}
& \sigma_{\mathrm{L}}=\sigma_{1}(\theta) \cdot \sin ^{2}(\varphi) \\
& \sigma_{\mathrm{T}}=\sigma_{1}(\theta) \cdot \cos ^{2}(\varphi) \\
& \tau_{\mathrm{LT}}=\sigma_{1}(\theta) \cdot \sin ^{2}(\varphi) \cdot \cos ^{2}(\varphi)
\end{aligned}
$$

The stress under consideration of the strength is here called "related stress" $B_{\sigma}$, and can be calculated with Eq. (7):

$$
|| \begin{gathered}
F_{1} \\
F_{2} \\
0
\end{gathered}|\cdot| \begin{array}{ccc}
F_{11} & F_{12} & 0 \\
F_{12} & F_{22} & 0 \\
0 & 0 & F_{66}
\end{array}|\cdot| \begin{gathered}
\sigma_{\mathrm{L}} \\
\sigma_{\mathrm{T}} \\
\tau_{\mathrm{LT}}
\end{gathered}|| \cdot\left|\begin{array}{c}
\sigma_{\mathrm{L}} \\
\sigma_{\mathrm{T}} \\
\tau_{\mathrm{LT}}
\end{array}\right|=B_{\sigma}
$$

with

$$
\begin{aligned}
F_{1}= & \frac{1}{\sigma_{\mathrm{LZ}}}+\frac{1}{\sigma_{\mathrm{LD}}}, \\
F_{11}= & -\frac{1}{\sigma_{\mathrm{LZ}} \cdot \sigma_{\mathrm{LD}}}, \\
F_{12}= & \frac{2}{\sigma_{45^{\circ} \mathrm{Z}}^{2}} \cdot\left[1-\frac{\sigma_{45^{\circ} \mathrm{Z}}}{2 \cdot\left(F_{1}+F_{2}\right)}\right. \\
& \left.-\frac{\sigma_{45^{\circ} \mathrm{Z}}^{2}}{4 \cdot\left(F_{11}+F_{22}+F_{66}\right)}\right], \\
F_{2}= & \frac{1}{\sigma_{\mathrm{TZ}}}+\frac{1}{\sigma_{\mathrm{TD}}}, \\
F_{22}= & -\frac{1}{\sigma_{\mathrm{TZ}} \cdot \sigma_{\mathrm{TD}}}, \\
F_{66}= & \frac{1}{\tau_{\mathrm{B}}^{2}},
\end{aligned}
$$

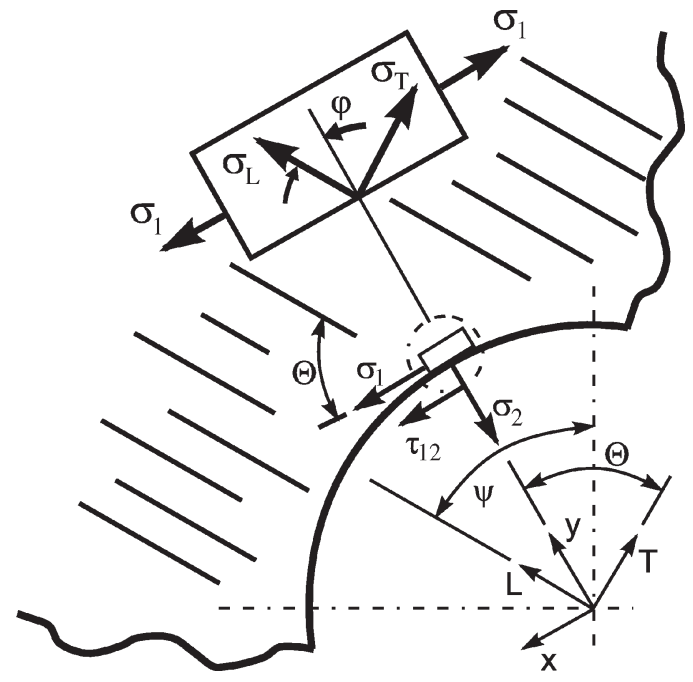

Fig. 7. System of co-ordinates along the hole contour.

where $\sigma_{\mathrm{L}}, \sigma_{\mathrm{T}}-$ stress in and perpendicular to the reinforcement direction, respectively, $\tau_{\mathrm{LT}}-$ shear stress within the fibre co-ordinate system $(\mathrm{L}, \mathrm{T})$.

This failure criterion needs the following material parameters which were determined experimentally:

$$
\begin{aligned}
& \sigma_{\mathrm{LZ}} \quad \text { - tensile strength in reinforcement direction, } \\
& \sigma_{\mathrm{TZ}}-\text { tensile strength perpendicular to the rein- } \\
& \text { forcement direction, } \\
& \sigma_{45^{\circ} \mathrm{Z}} \text { - tensile strength in diagonal reinforcement } \\
& \text { direction, } \\
& \sigma_{\mathrm{LD}}-\text { compression strength in reinforcement di- } \\
& \text { rection, } \\
& \sigma_{\mathrm{TD}}-\text { compression strength perpendicular to the }
\end{aligned}
$$

Figures 6(c) and (d) illustrate the related stress at the notch contour. The reinforcement influence on the related stress can be recognised by comparing the diagrams (a) with (c) and (b) with (d).

For both reinforcement-impact orientations, the stress in the reinforcement direction leads to very low related stress (diagrams (a), (c): at co-ordinates $90^{\circ}$ and $270^{\circ}$; diagrams (b), (d): at co-ordinates $45^{\circ}$ and $225^{\circ}$ ).

In case of the fibre direction of $0^{\circ}$, the maximum related stress occurs perpendicular to the reinforcement although the stress is very low (Fig. 6(a) and (c): at coordinates $0^{\circ}$ and $180^{\circ}$ ). Here the strength of composite is minimum. Contrary to the parallel fibre-impact orientation, within the specimen with the fibre orientation of $45^{\circ}$ the related stress perpendicular to the reinforce- 
ment is almost minimum (Fig. 6(d): at co-ordinates $135^{\circ}$ and $315^{\circ}$ ). Here, the maximum related stress occurs under approximate $45^{\circ}$ to the reinforcement at the co-ordinates $90^{\circ}$ and $270^{\circ}$, Fig. 6(d).

\section{Conclusions}

The experimental method presented here has proved to be good to visualise and analyse wave propagation and stress concentrations within impact loaded fibre reinforced composites. Moreover, the results clarify the dependence of wave propagation and spatial distribution of notch stresses on both reinforcement direction and time after impact. The investigations of uni-directional reinforced composite have shown that the maximum related stress does not generally occur perpendicular to the reinforcement direction. The direction of the maximum related stress varies within a range of $45^{\circ}$ to the reinforcement direction. Further- more, the amount of the related stress depends on the orientation of the reinforcement to the impact direction. Therefore, the maximum related stress after an impact loading can be minimised by an adequate fibre orientation.

\section{References}

[1] W. Hufenbach, M. Schäfer and A.S. Herrmann, Photoelastische Dehnungsmessung und Spannungsverteilung an faserverstärkten Bauteilen, Kunststoffe, 1991.

[2] K. Moser, Faser-Kunststoff-Verbund, VDI Verlag, Düsseldorf, 1992.

[3] A. Tenzler, Entwicklung einer Methode zur Ermittlung von Kerbwirkungen an impactbeanspruchten Faserverbundwerkstoffen, VDI Verlag, Düsseldorf, 1994.

[4] A. Tenzler and D.H. Müller, Rechnergestütztes Verfahren zur Bestimmung farbiger Isochromatenordnungen in der Spannungsoptik, Vision \& Voice Magazine 7(1) (1993), 34-39. 

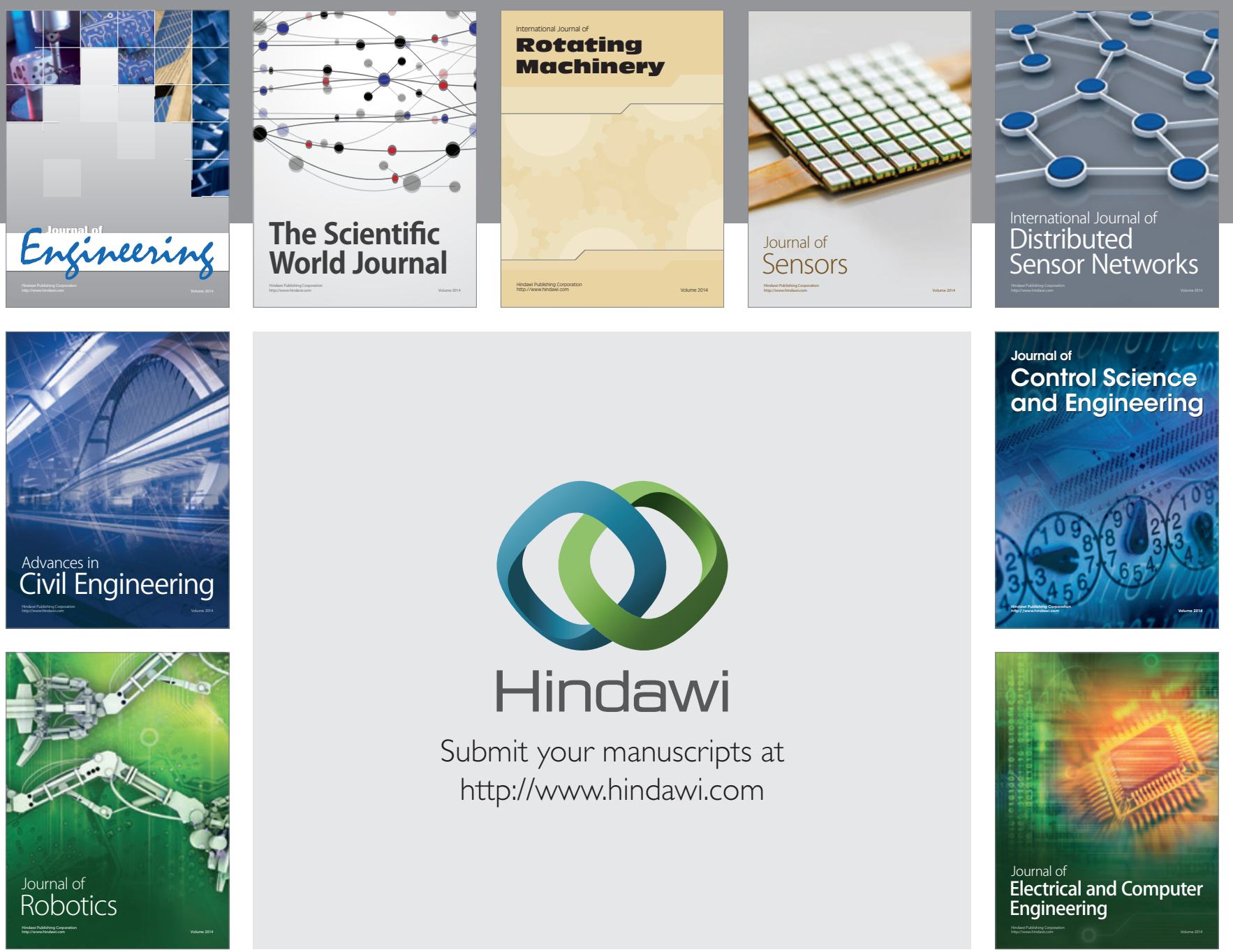

Submit your manuscripts at

http://www.hindawi.com
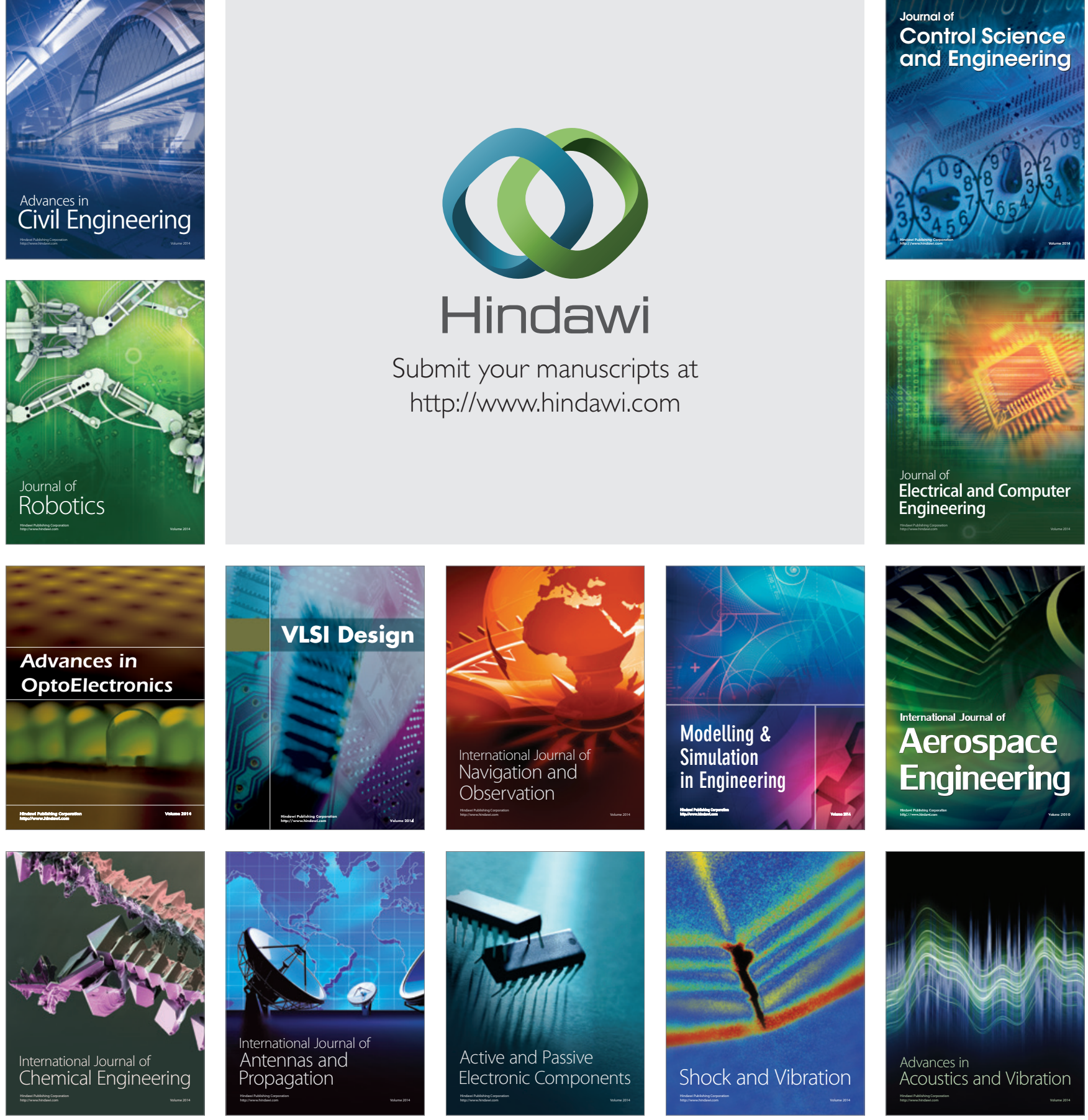